\title{
Les fantômes du Dendi
}

Lorsque surgissent les restes d'une ancienne filière textile au Nord Bénin The Phantoms of the Dendi (North Benin). When the Remains of an Ancient

Textile Sector Resurface

\section{Olivier Gosselain et Lucie Smolderen}

\section{(2) OpenEdition \\ Journals}

Édition électronique

URL : https://journals.openedition.org/tc/8198

DOI : $10.4000 /$ tc. 8198

ISSN : 1952-420X

Éditeur

Éditions de l'EHESS

\section{Édition imprimée}

Date de publication : 31 octobre 2016

Pagination : 452-469

ISBN : 9782713225291

ISSN : 0248-6016

Référence électronique

Olivier Gosselain et Lucie Smolderen, « Les fantômes du Dendi », Techniques \& Culture [En ligne], 65-66 2016, mis en ligne le 31 octobre 2018, consulté le 07 décembre 2022. URL : http:// journals.openedition.org/tc/8198; DOI : https://doi.org/10.4000/tc.8198 


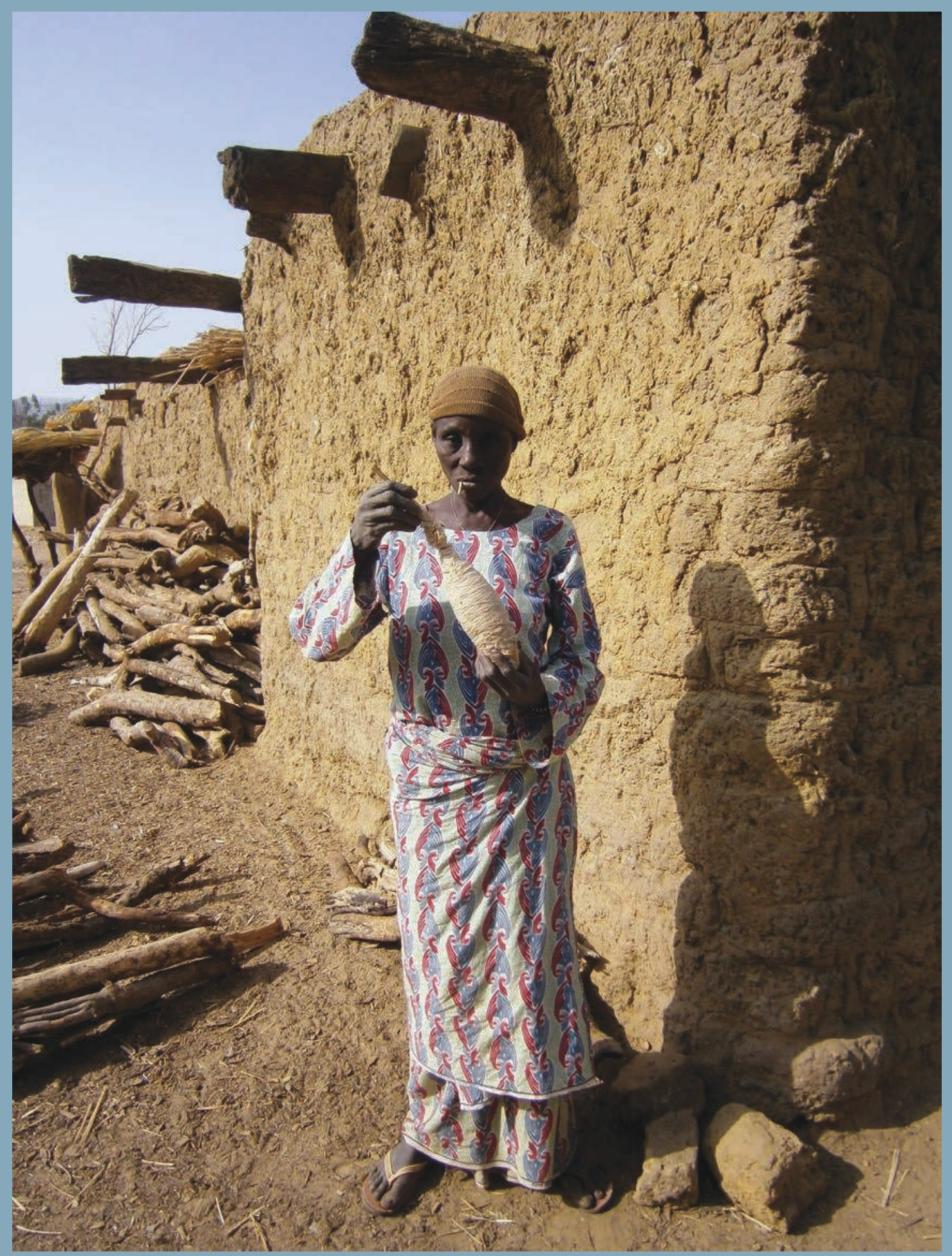




\section{Les fantômes du Dendi}

\section{Lorsque surgissent les restes d'une ancienne filière textile au Nord Bénin}

What moron said the past is dead? The past is not dead. Its phantoms own us. Siri Hustvedt

En février 2011, Olivier effectuait un inventaire des artisanats et de la culture matérielle dans le Dendi béninois, pour le compte du projet Crossroads of Empires ${ }^{1}$ (Haour et al. 2011). Associées à un programme de fouilles archéologiques, les enquêtes ethnographiques portaient principalement sur la métallurgie et la poterie, mais quelques entretiens avaient lieu avec d'anciens tisserands, en raison de leur association fréquente avec les potières. Au cours d'un de ces entretiens, une activité s'invita soudain dans la conversation, qui allait occuper une place croissante dans nos enquêtes, jusqu'à supplanter les deux autres. Les tissus en coton, expliquait un tisserand ${ }^{2}$, étaient autrefois teints «en jaune, rouge ou noir. » Lactivité de teinture était surtout développée à Kano (Nigeria), ajoutait-il, mais on l'avait également pratiquée non loin de là, à Karimama, dans un quartier où subsistaient les restes d'un ancien atelier.

Une visite de ce quartier permit d'identifier quelque vingt-cinq puits comblés de déchets et fortement érodés. Elle révéla également l'existence de quelques anciens praticiens prêts à nous renseigner sur leur métier. L'entretien mené avec l'un d'eux ${ }^{3}$ allait apporter un fait supplémentaire: il paraissait envisageable de documenter une activité disparue sur bases de témoignages oraux.

Ce surgissement inattendu d'un passé technique donné pour mort fut suivi de situations similaires lorsque Lucie se lança en 2012 dans l'étude de la filière textile artisanale. Des navettes de tisserand, des fuseaux, des cardes, des pierres à égrener émergeaient spontanément en cours d'enquête (Smolderen \& Minguet 2014). De vieilles femmes étaient surprises en train de filer dans leur concession. Bien que la production textile se soit éteinte plusieurs décennies auparavant et sembla reléguée à l'arrière-plan des préoccupations sociales et historiques de nos informateurs, certains de ses constituants refusaient manifestement de disparaître. Mais ils le faisaient en ordre dispersé, ce qui nous forçait à considérer le processus de disparition d'une 
filière technique dans toutes ses nuances. Un nouveau volet d'enquête venait de s'ouvrir (Haour et al. 2011, Smolderen 2013, Smolderen \& Minguet 2014).

Dans cet article, nous ne traiterons pas de l'objectif ultime de ce volet d'enquête: la reconstitution aussi minutieuse que possible des chaînes opératoires de l'ancienne filière textile et leur comparaison afin d'enrichir la compréhension historique du Dendi béninois. Compte tenu du thème de ce numéro, nous avons choisi de nous intéresser aux restes associés à cette ancienne filière et à la façon dont ils travaillent la société Dendi contemporaine et nos propres recherches.

\section{Ce qui reste quand rien n'est censé rester}

Les restes considérés ici sont des fantômes et non des survivances. Ces dernières sont inévitables, puisque le paysage technique ou matériel d'une société ne relève jamais du palimpseste. Même dans un monde dominé par l'obsession de progrès, l'ancien côtoie toujours le nouveau - parfois au sein des mêmes dispositifs techniques (Jacomy 2002: 15-28). Qu'une poignée de vieilles femmes du Dendi continuent à filer un coton glané au bord des routes alors que la filière textile artisanale s'est effondrée n'a rien d'étonnant. Comme les potières de la région, elles sont les dernières représentantes d'une activité appelée à disparaître avec elles.

Parler de «fantômes», c'est évoquer au contraire ce qui refuse de disparaître lorsque l'activité est morte; ce qui revient hanter un présent qui paraissait en être quitte. Il s'agit en l'occurrence de différentes catégories de restes qui ont, comme les techniques et les objets « vivants» (Gell 1992, Julien \& Rosselin 2009), la capacité d'agir sur, et de faire agir, les personnes, quels que soient les liens qu'elles entretiennent avec l'activité qui les produit. Nous verrons ainsi comment ces restes affectent d'anciens praticiens, mais aussi d'autres catégories de sujets, dont les chercheurs.

Leur caractère hétéroclite nous a forcés d'emblée à coller au plus près des évocations et des situations d'apparition. Nous continuerons à le faire ici. En prêtant attention à la visibilité physique et sociale de ces «restes fantômes", ainsi qu'aux cadres de pratiques auxquels ils sont associés, nous verrons qu'il est possible de les regrouper en catégories et d'explorer leurs effets contrastés sur le présent.

\section{Irréductibilité}

Lactivité technique et les restes qu'elle produit ont différentes façons d'être là sans plus y être. Le coton en est un bon exemple: toujours présent et bien visible dans le Dendi, il a été absorbé depuis les années 1960 par une filière de production sans rapport avec l'ancienne. Qu'il s'agisse des quantités produites, des techniques agraires, des modes de récolte, des acteurs, des finalités 


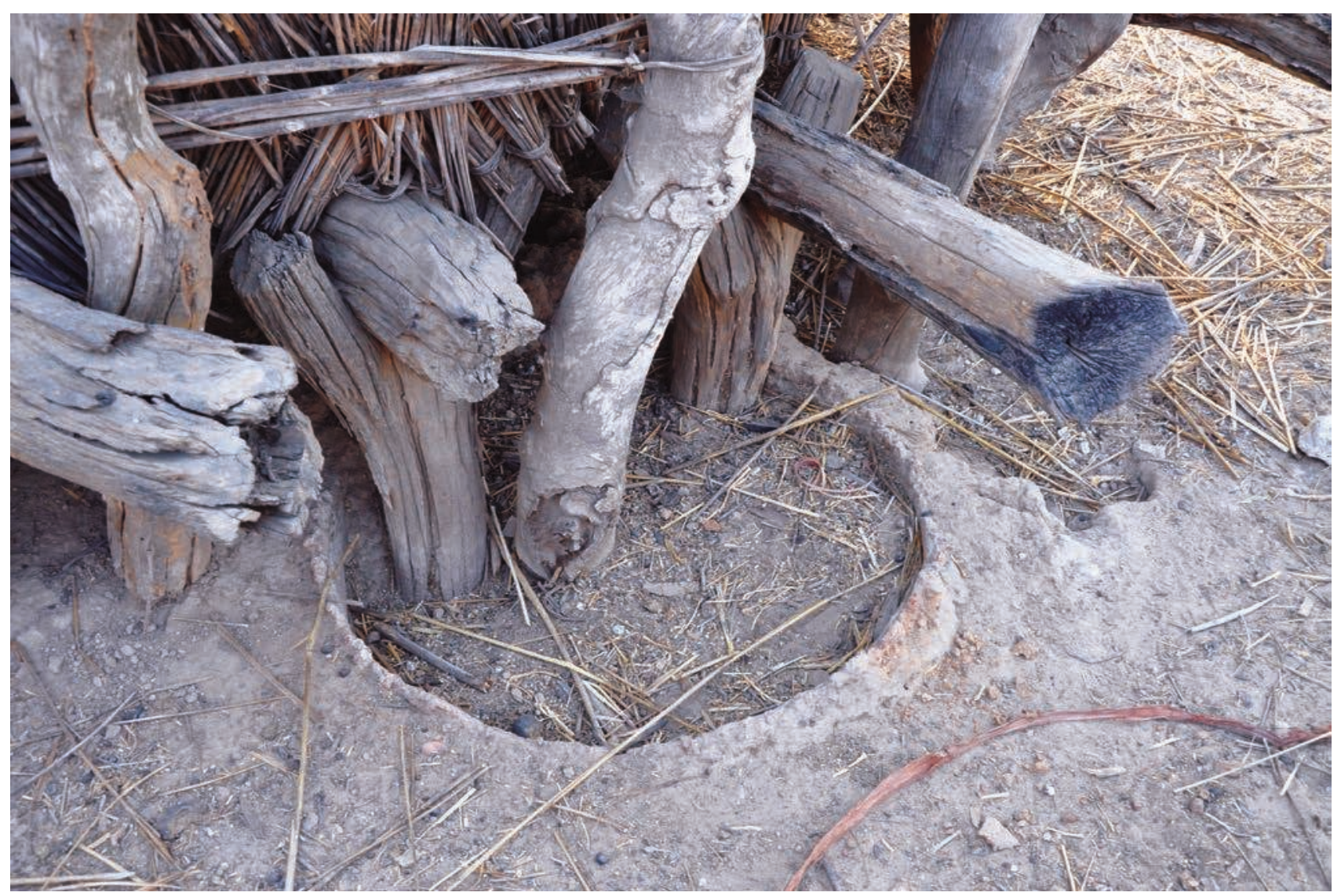

et même de son identité génétique, plus rien ne raccorde le coton actuel à son passé (Smolderen 2013). La rupture se marque alors par un mode de présence plutôt qu'une absence.

Une autre forme d'absorption est le recouvrement. Bon nombre d'anciens ateliers de teinture à l'indigo ont été lotis, leur existence se marquant désormais sur le sol par quelques cercles blanchâtres (qui correspondent aux parois des cuves), dans lesquels s'enfonce parfois un poteau de soutènement ou un mur de fondation.

Tout en conservant une certaine visibilité, le passé technique semble ainsi sagement descendre dans le sous-sol des vivants.

Toutefois, les caractéristiques mêmes de la technique de teinture à l'indigo ont la capacité de perturber cet ordonnancement stratigraphique. Hormis les feuilles d'Indigofera, une matière première essentielle est en effet la boue résiduelle extraite des cuves après la vidange de la teinture usagée. Calciné dans un foyer de branches fraîches et pilé avec la cendre pour en obtenir une fine poudre, ce matériau est aussi bien utilisé dans la construction et la réfection des cuves que dans la préparation de la teinture. 
2. Sur le site de l'ancien village de Bwayeri, détruit dans les années 1880, un monticule de sédiments blanchâtres marque l'emplacement d'un atelier de teinture à l'indigo. Ramené à la surface par les activités agricoles, ce matériau facilement interprétable contribue au maintien d'une mémoire technique depuis la fin du xIxesiècle.

3. Banikoara, quartier Maréworou. Dans cette localité située au sud de notre zone d'étude, le dernier teinturier en activité s'apprête à calciner la boue résiduelle d'une cuve à indigo. Sur le pourtour de son atelier, transformé en conservatoire, il cultive différentes plantes tinctoriales dont on se servait par le passé.
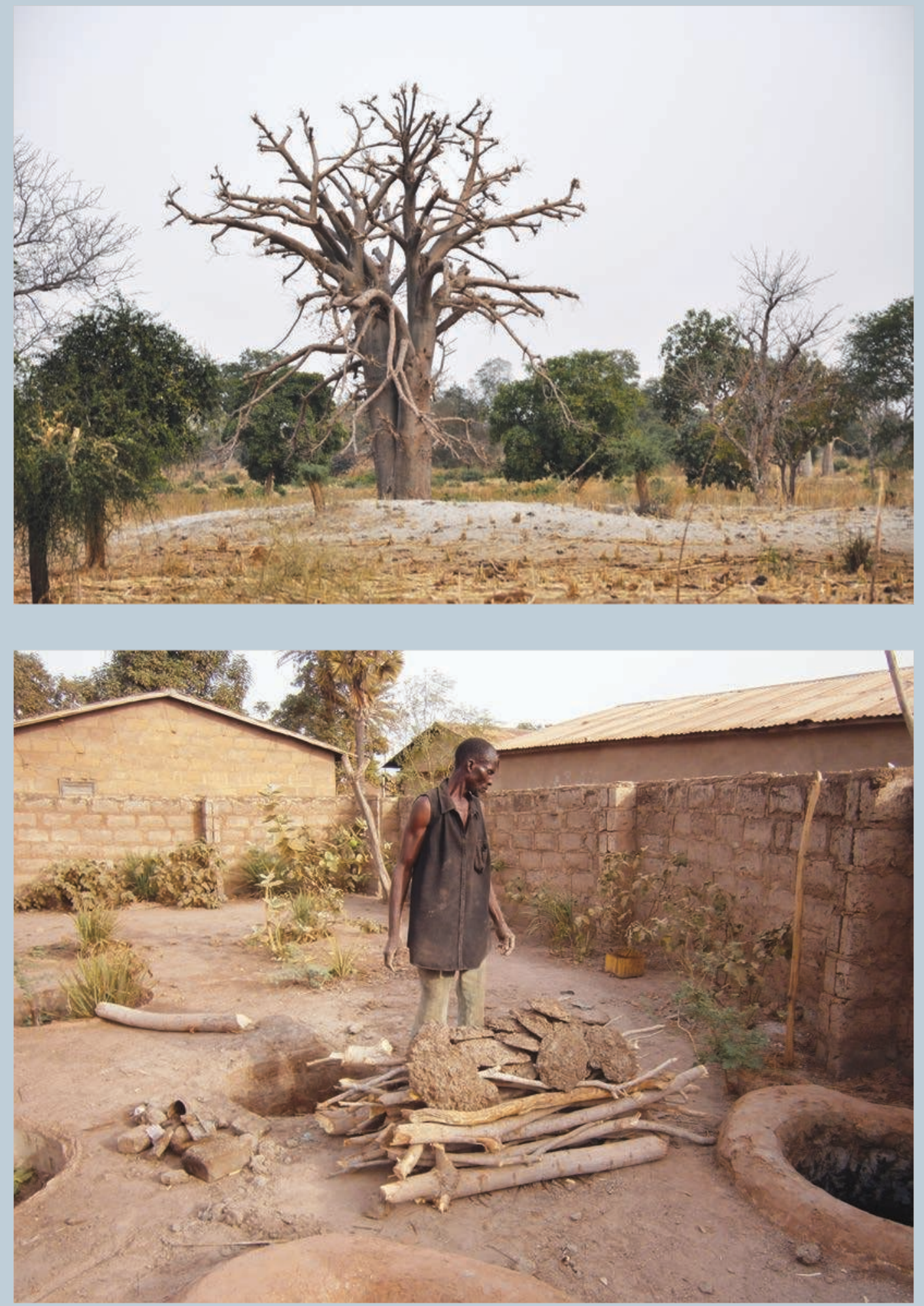
Dans le premier cas, le matériau est humidifié et appliqué comme un ciment sur les parois. Dans le second, il est ajouté en quantités variables ${ }^{4}$ à la préparation, où son rôle est comparable à celui de la potasse. Du point de vue de la pratique vivante de l'activité, la boue résiduelle apparaît ainsi comme un reste irréductible et vital. Irréductible, parce qu'elle sort inlassablement des cuves pour y revenir transformée ou donner naissance à de nouvelles cuves; vitale, car les praticiens la considèrent comme un ingrédient indispensable à la réussite des opérations, et sans alternative connue.

Mais les boues calcinées sont également des fantômes. Présentes en quantité dans les ateliers abandonnés, elles prennent l'apparence d'amas de fins sédiments blanchâtres lorsque le sol est perturbé par les activités agricoles ou les animaux fouisseurs. C'est le cas à Bwayeri, un ancien village incendié et abandonné dans les années 1880, suite à une guerre qui marque encore la mémoire régionale. Lors d'une première visite du site, nos accompagnateurs montrent cet amas et l'interprètent comme un ancien atelier de teinture.

C'est néanmoins le seul vestige qui fasse l'objet d'une interprétation de leur part. Symptomatiquement, les traditions orales relatives au site évoquent la teinture à l'indigo, mais aucune autre activité de production: on ne sait rien de la métallurgie, de la poterie ou du tissage à Bwayeri, mais on sait qu'on y pratiquait la teinture et que cette activité fut probablement à l'origine de la prospérité du village. Difficile de ne pas voir à l'œuvre l'effet d'«irréductibilité» des amas cendreux: plus visibles et sémiologiquement plus transparents que d'autres restes, ils orientent la compréhension du passé et les narrations de nos interlocuteurs. Leur effet se fait également sentir sur les archéologues. Ayant acquis le statut de vestige durable et interprétable, les amas cendreux ont capturé l'attention depuis 2012: on les recherche dans les sondages; on croit les avoir trouvés lorsque la truelle pénètre dans une couche plus claire; on s'interroge sur les instruments ou les mesures qui en révéleraient la présence depuis la surface... Sans aucun succès jusqu'ici.

Une autre catégorie de restes irréductibles existe dans le Dendi, qui ne relève pas du domaine matériel. Comme dans bien d'autres régions d’Afrique (Olivier de Sardan 1984, Tamari 1997), le Dendi précolonial connaissait une opposition fondamentale entre personnes libres et esclaves. Parmi les esclaves figuraient des hommes (et des femmes) «à tout faire», mais aussi plusieurs groupes socioprofessionnels spécialisés, comme les potières et les tisserands. Quoique l'esclavage ait été aboli il y a plus d'un siècle et que le tissage ne soit plus pratiqué depuis trente ans au moins, les termes «tisserand » et «esclave» restent non seulement présents dans les discours, mais également indissociables. À propos d'une chanson que l'on destine aux tisserands lors des cérémonies de mariage et de baptême, l'un de nos interlocuteurs ${ }^{5}$ précise:

«À côté des chants que l'on chante en travaillant, il y avait aussi des chants et des danses que les [griot] chantaient aux tisserands lors de fêtes [...]. C'est la chanson Caakey de Caakey [Tisserand Ô Tisserand]. Encore aujourd'hui, quand un fils de tisserand se marie, on lui chante ça, et tout le monde sait que c'est un fils d'esclave ${ }^{6} . »$ 
4. L'ancien atelier de teinture de Karimama en février 2012.

Après nettoyage et construction d'un mur de protection. II ne reste rien de cette clôture aujourd'hui.
Or, le «fils du tisserand» évoqué ici n’a jamais pratiqué l'activité, ce qui ne l'empêche pas d'être associé à son père et à un statut dévalorisé. Enchâssés, l'activité technique et le statut social demeurent constitutifs de l'identité des hommes de la famille.

Un fils de tisserand qui souhaite se marier doit ainsi demander le consentement de ceux dont sa famille dépendait autrefois ${ }^{7}$. Il s'agit d'un avatar contemporain du contrôle matrimonial que les maîtres exerçaient sur leurs esclaves (Olivier de Sardan 1984).

On peut s'étonner de l'insolente continuité de ce statut, que tant préféreraient voir disparaître. Derrière son immatérialité, l'idéologie esclavagiste (Olivier de Sardan 1982) qui sous-tend ces rapports sociaux n'a rien de spectral. Au contraire, comme Bornand (2011) l'a montré pour les sociétés songhay-zarma du Niger, la dichotomie entre nobles et captifs n’a jamais disparu dans le Dendi, malgré l'extinction des activités dont elle structurait le fonctionnement social. Une des raisons tient sans doute au fait que cette dichotomie se déployait bien au-delà des activités artisanales dont il est question ici. Dans le cas des tisserands, il nous semble néanmoins qu’un autre élément pourrait

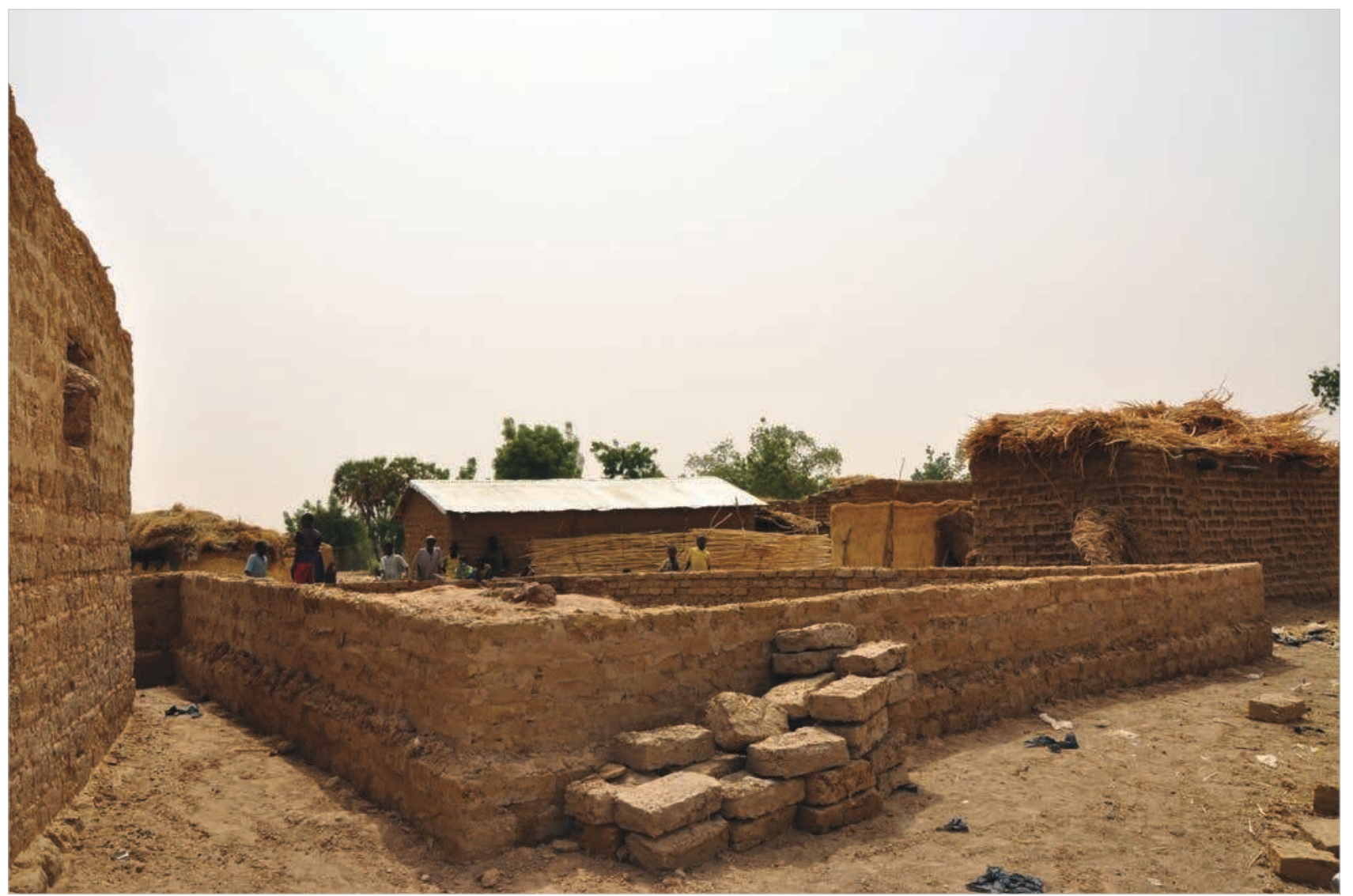


être pris en compte. Prenons l'exemple de la chanson évoquée plus haut. À son propos, un informateur d'Olivier de Sardan expliquait dans les années 1970 que «si on bat cet air-là tout le monde sait qu'il y a des [esclaves] » (1984: 55).

Quarante ans plus tard, le parallèle est frappant: malgré les transformations du monde contemporain, une association persiste entre le chant et le statut social des tisserands. Or, cette persistance semble assurée par les dispositifs dans lesquels sont pris les tisserands, hors de leurs activités de tissage. Le griot, le rythme battu sur le tambour, l'air de la chanson, ses paroles reprises par l'assistance, la danse qui l'accompagne ${ }^{8}$, sans oublier le fils du tisserand, objet de l'attention collective et descendant d'une lignée connue: tous ces éléments mis en relation lors des cérémonies créent un « dispositif scénique » (Hénnion 1988: 11) servant de point d'ancrage à ce statut immatériel et irréductible. Celui-ci existe alors au travers d'une série de médiations (Hénnion 1988, Legrain 2009), parmi lesquelles la chanson «Tisserand Ô Tisserand » semble être un véhicule circonstanciel qui en assure la continuité dans un monde en transformation.

\section{Valorisation}

Les restes qui viennent d'être évoqués hantent les

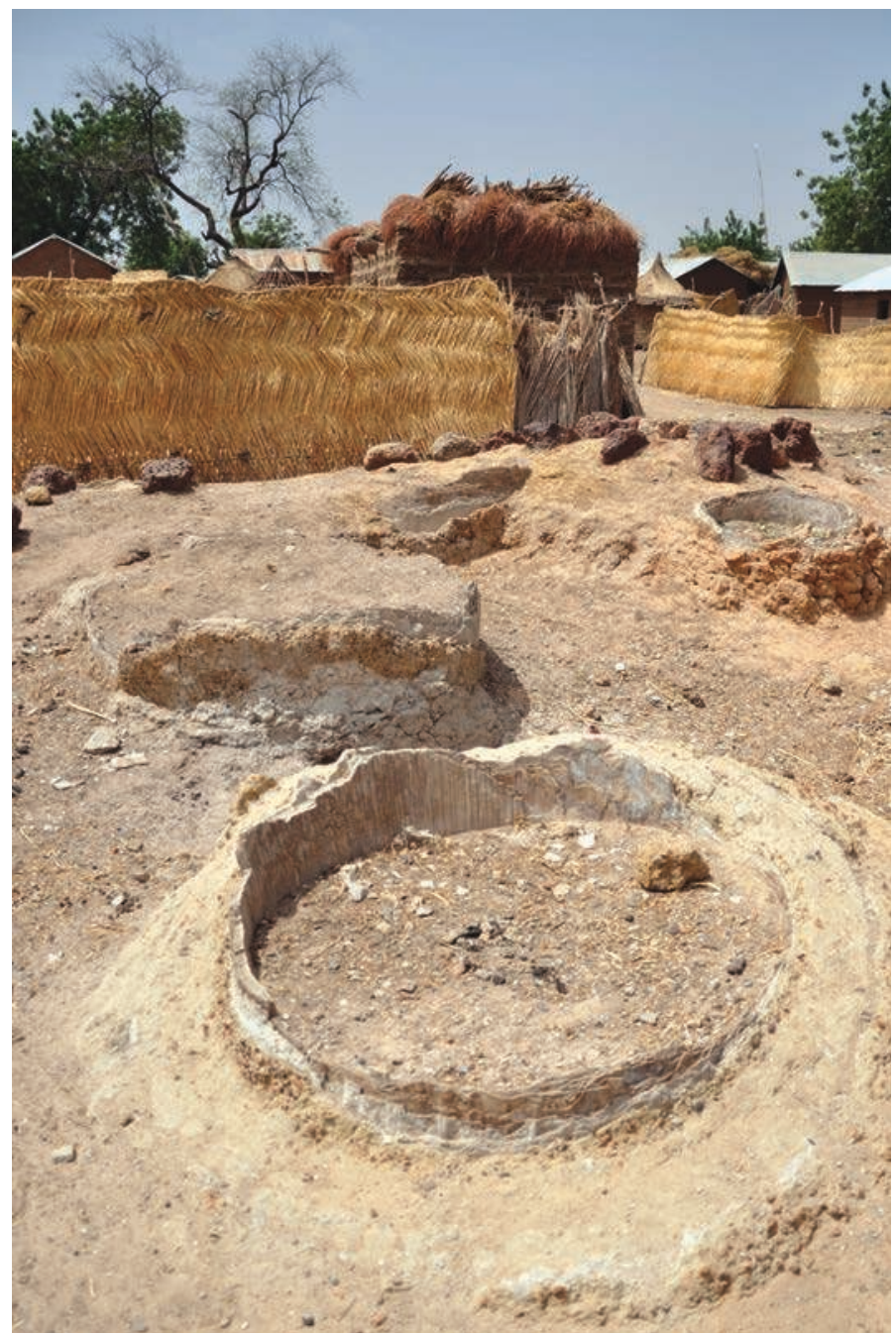
vivants malgré eux - pour le meilleur et pour le pire-en raison de qualités intrinsèques ou de la persistance des médiations qui leur servent de support. Il en est d'autres, cependant, dont la présence et le surgissement occasionnel résultent d'une démarche délibérée de conservation, que l'on qualifierait volontiers - paresseusement?de «patrimoniale» ou «mémorielle.» Notre première confrontation avec un tel phénomène eut lieu sur le site de l'atelier de teinture de Karimama.

L'homme qui nous y avait accueillis était un pêcheur d'une soixantaine d'années résidant dans une concession voisine. Il avait tout de suite fait part de ses inquiétudes quant au devenir du site. L'endroit était érodé par les pluies, les riverains y jetaient des détritus et l'utilisaient comme latrines et, surtout, on souhaitait y construire des habitations. Or, nous disait-il, les

5. Karimama, quartier Batouma Beri

L'ancien atelier de teinture à l'indigo en février 2011. Utilisé comme dépotoir et menacé par l'extension des habitations, sa survie ne tenait à l'époque qu'à la volonté opiniâtre d'un notable du quartier. 
cuves étaient les témoins de l'ancienne activité des hommes du quartier. Elles étaient aussi les mieux conservées de la région ${ }^{9}$. Il fallait donc les protéger pour pouvoir montrer aux enfants et aux visiteurs comment s'effectuait autrefois le travail de la teinture. À ce stade, nous ignorons encore si les velléités patrimoniales de cet homme - et des élus locaux qui les relayaient - constituaient le seul ressort de ce qui a toutes les apparences d'un conflit foncier. Elles avaient en tout cas contribué à préserver un site explicitement investi d'une valeur mémorielle.

Une telle démarche était sans doute d'autant mieux accueillie que la teinture à l'indigo appartient à un passé valorisé. C'était une activité d'homme libre et l'une des seules-avec le commerce des chevaux - qui permettait de s'enrichir honnêtement.

«Les teinturiers étaient fiers de leurs mains noires», nous dit un informateur ${ }^{10}$. «Après s'être lavés, ils mettaient du beurre de karité dessus pour les faire briller. Ils se reconnaissaient entre eux au marché.»

La teinture offrait par ailleurs des possibilités de recyclage aux vieux qui n’avaient plus la force de travailler aux champs - situation difficilement envisageable aujourd'hui. Du côté des membres du projet de recherches Crossroads of Empires, le discours patrimonial de nos interlocuteurs avait trouvé un écho immédiat, tant en raison des finalités historiques du projet que de l'intérêt des bailleurs de fonds pour les retombées sociétales de la recherche. Préserver et valoriser un site auquel tenaient «les populations locales» était une excellente démarche en termes de relations publiques. C'est ainsi qu'en 2012, l'ancien atelier fut nettoyé et entouré d'un mur de briques de terre crue.

On évoquait aussi la construction d'une toiture en tôle et la réhabilitation d'une des cuves pour l'année suivante, en vue d'une valorisation touristique. Las! Le tourisme est un véritable Désert des Tartares dans le Dendi. Et l'on n'investit pas impunément de l'argent dans la construction d'un mur... L'initiative s'est enlisée et au moment d'écrire ces lignes, le mur a disparu, les cuves sont un peu plus dégradées, le site a repris une fonction de dépotoir. Les restes entrent ainsi en repos, attendant peut-être une nouvelle occasion de surgir.

D’autres formes de conservation délibérée ont été observées sur le terrain. Moins impressionnantes que l'atelier de Karimama du point de vue de leurs effets, elles suivent néanmoins une même logique. À Mamassi Gourma, un ancien teinturier nous entraîne dans un de ses champs, en bordure duquel poussent quelques plans d'Indigofera tinctoria.

Il explique les y avoir semés il y a plus de dix ans pour pouvoir les montrer à tout visiteur intéressé par le passé teinturier de son village. À Kompa, ce sont trois vieilles qui exhibent une bande de coton jaunie en cours d'entretien.

«Pourquoi l'avoir conservée?», demande Lucie.

«Pour la mémoire!», répond l'une d'elles.

On trouve ici l'écho d'une situation observée par Ravenhill (1991) en Côte d'Ivoire, dans laquelle une démarche muséographique est adoptée par un individu soucieux de préserver le passé de sa communauté. Ce double projet de préservation et de transmission peut prolonger des pratiques qui avaient cours lorsque l'activité existait encore. Certains outils associés aux chaînes opératoires du tissage et du filage s'inscrivent ainsi dans une dynamique d'héritage familial. Au terme d'un 


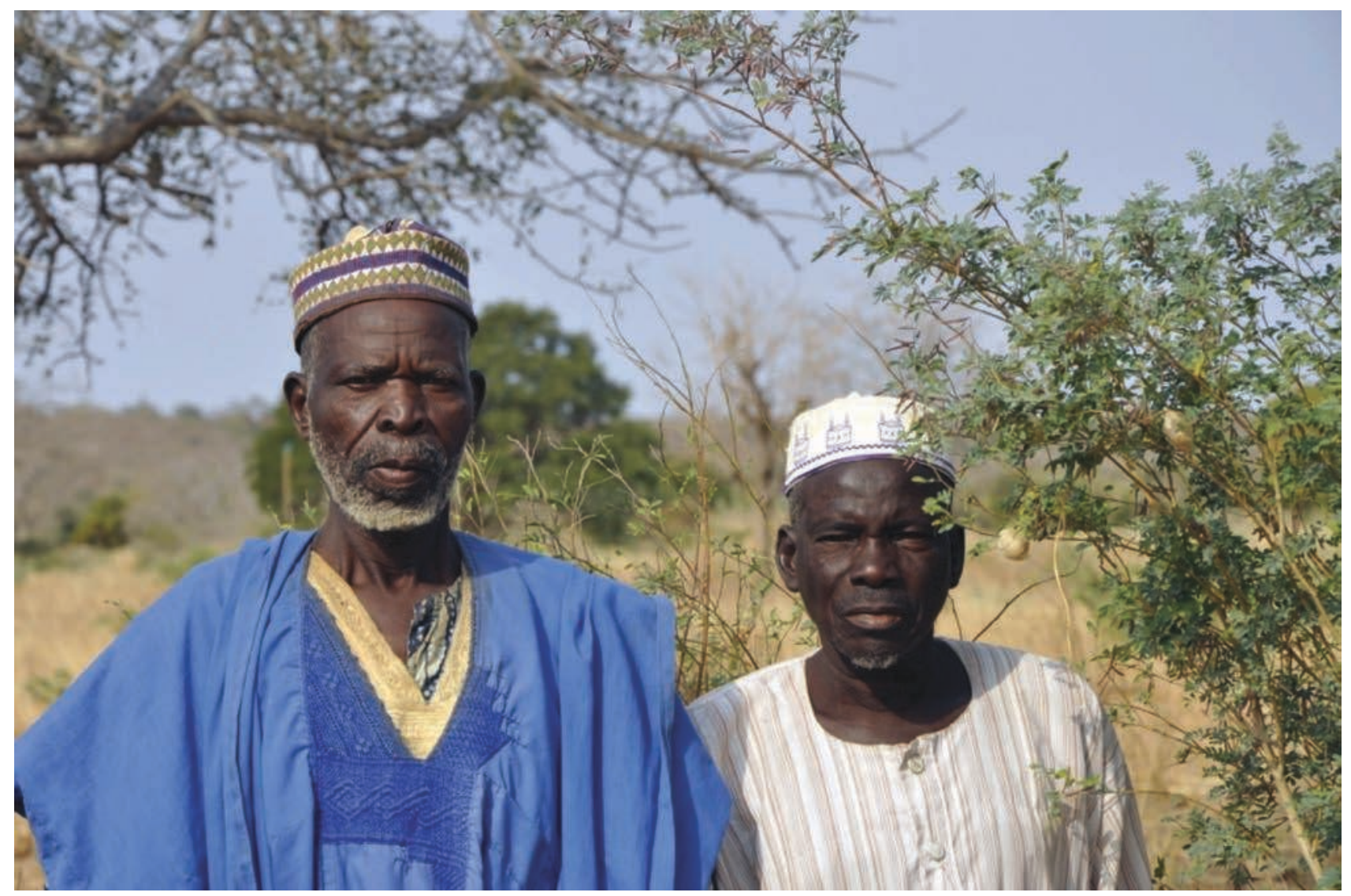

entretien avec un ancien tisserand ${ }^{11}$, son petit-fils rattrape Lucie, pour lui montrer deux navettes couvertes de purin qu'il venait de sortir de sa chambre. Quoiqu'il n'ait jamais pratiqué l'activité, il conservait les outils de son père. À l'époque de ce dernier, la navette et le peigne étaient transmis de père en fils. Un jeune tisserand travaillait avec les outils de son père avant d'obtenir les siens ${ }^{12}$. La volonté de conservation de cet objet est ainsi héritée - comme lui - du système technique disparu.

Le même phénomène touche deux objets manipulés par les fileuses: la pierre et la tige à égrener. Les femmes se les procuraient difficilement et toutes n'en possédaient pas ${ }^{13}$. Si leur prêt était fréquent, l'héritage permettait néanmoins de les conserver au sein de la lignée matrilinéaire.

Ces objets ont perdu aujourd'hui leur raison d'être technique. Ils sont en bout de course:

6. Deux anciens teinturiers de Mamassi Gourma à côté d'un plant d'Indigofera tinctoria (à droite).

L'un d'entre eux l'a planté dans son champ comme témoin d'une activité qui assurait la vitalité économique du village jusqu'au milieu du xxe siècle. les navettes en bois sont la proie des termites; la pierre s'émousse et va disparaître ${ }^{14}$; séparée de sa tige, les deux finiront par s'égarer. Mais si son heure est proche, la démarche mémorielle subsiste encore, comme en témoigne la présence de ces objets dans les foyers des descendants de praticiens. Le fantôme prend ici une figure de mémoire familiale. Hantant les stratégies patrimoniales, il vient s'opposer à la disparition «naturelle» de ces objets. 


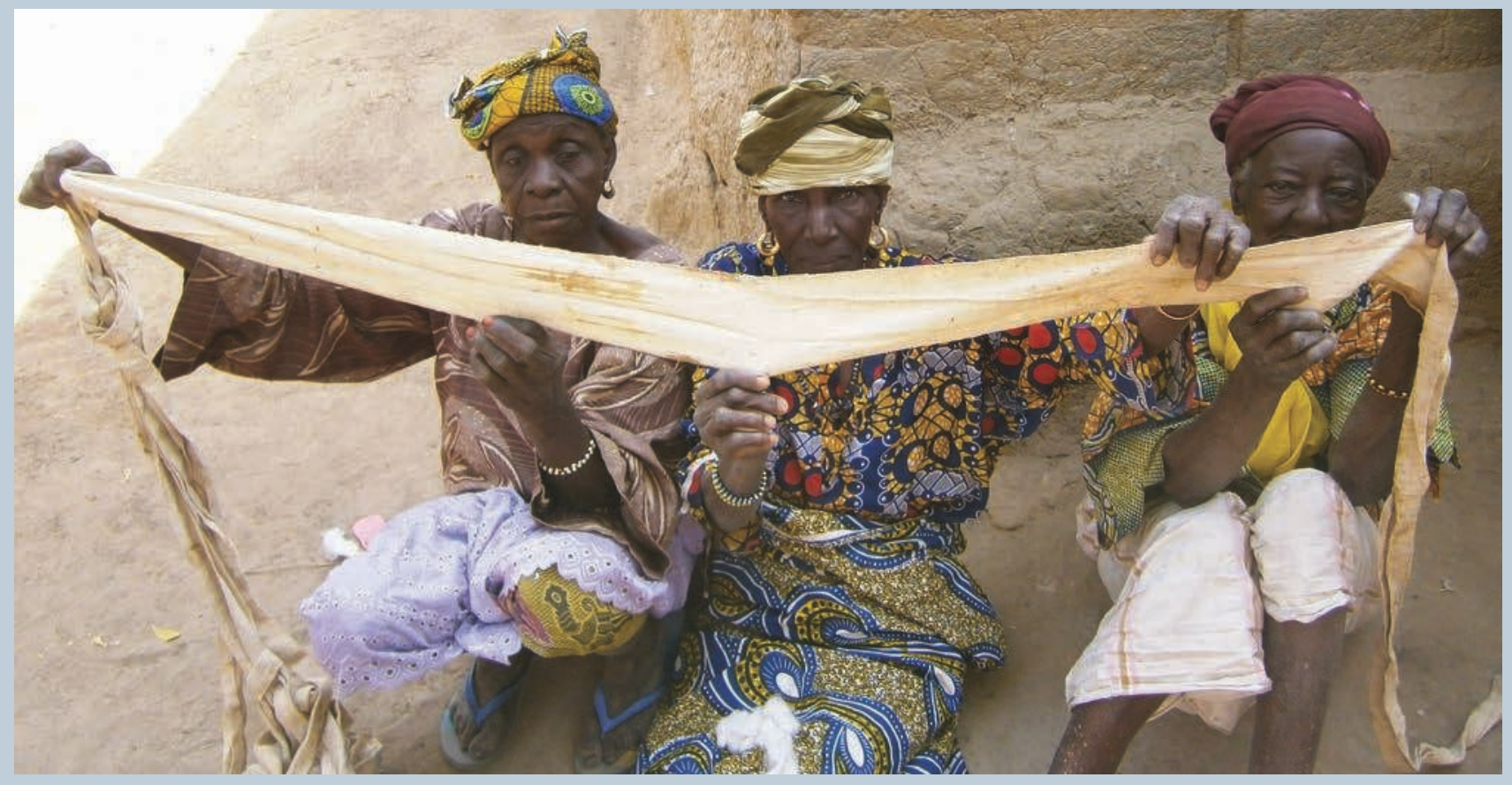

7. A la suite d'un entretien, nos interlocutrices exhibent fièrement une bande de coton jaunie, vieille d'une trentaine d'années. L'une d'elles a décidé de la conserver pour garder la mémoire de la filière textile du Dendi, aujourd'hui disparue. 


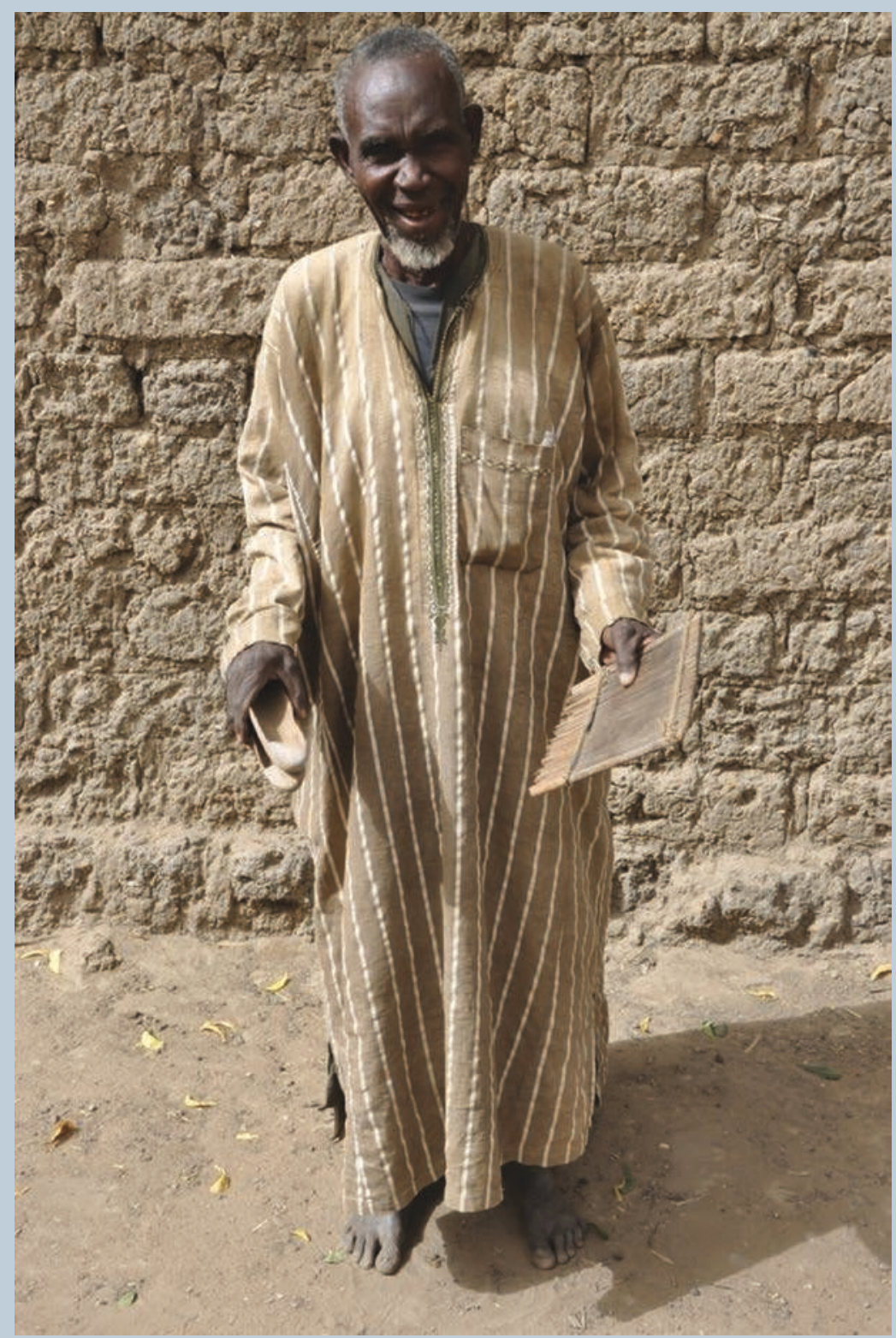

8. Un ancien tisserand nous montre ses vieux outils: deux navettes et un peigne, loin d'être intacts. Par le passé, ces objets étaient hérités de père en fils. Bien après l'arrêt de l'activité, la volonté de conservation persiste et s'appuie sur une même logique d'héritage. 


\section{Attachement impassible}

D'autres objets ont fait leur apparition lors d'entretiens avec des femmes âgées: fuseaux, vieille paire de cardes, bobines de fil poussiéreuses (Smolderen et Minguet 2014).

Tous sont liés à la chaîne opératoire du filage. Mais à l'inverse du dispositif d'égrenage, cardes et bobines n'ont jamais été des objets «hérités». On les retrouve aujourd'hui dans un coin de la chambre, sous le lit ou au fond d'un sac en plastique, pas toujours accessibles et non investis d'une charge mémorielle explicite. Inanimés, sans futur déterminé, ils sont remisés.

À l'époque où la filière artisanale du textile était encore active, le filage était une des activités principales des femmes.

«Les seuls jours de l'année où l'on ne filait pas, c'était quand il y avait des funérailles. C'est pourquoi tous les matins, on allait demander aux vieux si quelqu'un n'était pas mort durant la nuit. ${ }^{15}$ »

Les femmes d'une concession, et parfois quelques voisines, se réunissaient pour travailler ensemble. Les fillettes apprenaient suivant une trajectoire classique (Lave \& Wenger 1991) passant graduellement de l'observation à la participation au travail des aînées. C'est en entrant progressivement en corps à corps avec les outils et les matières que la jeune fille devenait fileuse. Mais comme le rappelle une de nos informatrices, le filage n'était pas seulement un moment pour «causer et chanter ${ }^{16}$, c'était également un travail lent et difficile $^{17}$. La jeune apprentie, dont les doigts, encore délicats, n'étaient pas habitués au fuseau, incorporait deux qualités essentielles de la «bonne épouse » au cours de son apprentissage: la patience et la capacité au travail. Pratiqué au quotidien dans le cadre familial, le filage était un moment de socialisation au cours duquel se construisait l'une des facettes de l'identité féminine. Les outils entraient quant à eux dans la composition du trousseau de la jeune mariée, aux côtés des ustensiles de cuisine. La pratique du filage et les objets qui y étaient associés contribuaient ainsi à la construction d'un ethos féminin, aux multiples facettes (voir à ce sujet Naji 2009, Vallard 2012).

Après l'arrêt de l'activité, les outils de filage n'ont pas été investis d'un projet mémoriel ou patrimonial. Ils «traînent» simplement là, dans les chambres des vieilles femmes. Comment comprendre cette inertie? Comment expliquer qu'ils soient conservés comme par défaut? D'abord, les caractéristiques physiques de ces outils les font facilement oublier. Dans un coin ou sous le lit, ce sont des fantômes discrets qui n'entravent ni ne menacent le quotidien. Par ailleurs, les cardes, le fuseau et les bobines sont des outils spécialisés dont les potentialités ne facilitent visiblement pas la requalification: ils n'ont pas pu s'insérer dans une autre filière technique pour y disparaître ou faire complètement oublier leur ancien statut. Alors ils restent, maintenus par une sorte d'attachement impassible. Leur présence n'est pas tant celle de résidus matériels d'une chaîne opératoire morte, que de fantômes d'un ethos féminin aujourd'hui en transformation.

À cet égard, l'absence d'un phénomène similaire chez les anciens teinturiers est étonnante. Aucun outil n'a pu être montré lors des enquêtes et il a fallu bien des efforts pour en 
dresser l'inventaire ou en préciser les caractéristiques. Leurs possibilités de requalification sont un facteur d'explication. Paniers, calebasses ou maillets en bois ont pu facilement trouver place dans d'autres activités et y disparaître. Il y a aussi leurs dimensions, qui s'opposent, plus que les cardes ou les fuseaux, à un entreposage discret. C'est certainement le cas des perches à croisillons servant à brasser la teinture ou des filtres à potasse. Toutefois, on ne peut s'empêcher de penser que la façon qu'ont les hommes libres de s'engager dans une activité comme la teinture n'est pas identique à celle des femmes dans le filage ou des esclaves dans le tissage. Sans nier les possibilités de constitution d'un ethos masculin dans l'activité de teinture, la principale dimension mise en avant dans les entretiens est le revenu économique, ce qui relève d'autres formes d'attachement. Lorsque le travail ne rapporte plus, on l'abandonne, on passe à autre chose ( $c f$. Gosselain 2015). Les restes pourraient alors être évacués sans état d'âme.

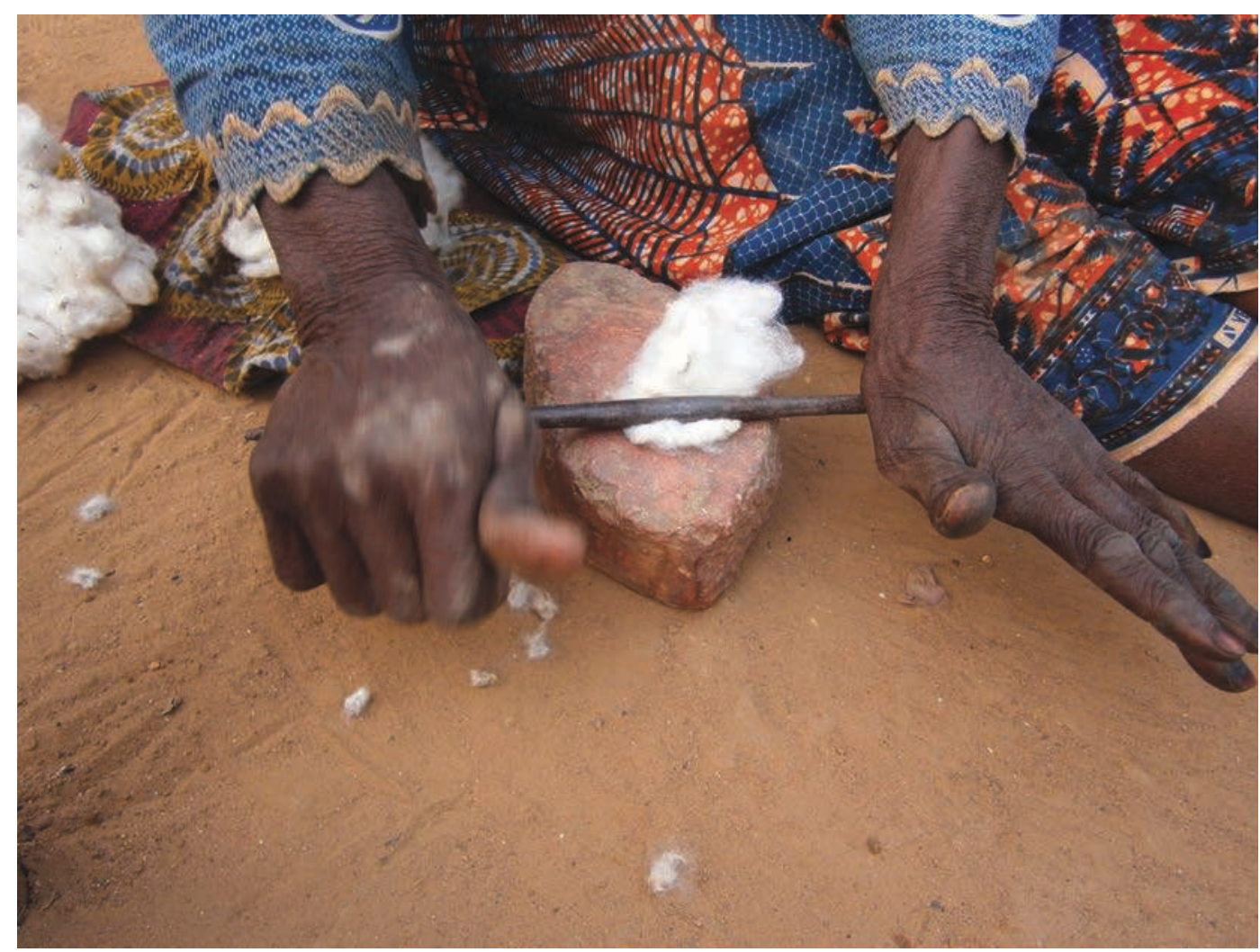

9. Une ancienne fileuse ressort une pierre, une tige à égrener et se lance dans une démonstration. Dans sa famille, ces outils sont transmis de mère en fille depuis plusieurs générations et elle les conserve précieusement, quoiqu'elle ne file plus. 
10. Dans le coin d'une chambre, cette vieille bobine - encore sur son support de travail - n'attend plus rien. Sans projet, sans utilité, rien ne lutte explicitement contre sa disparition naturelle, si ce n'est d'avoir été remisée.

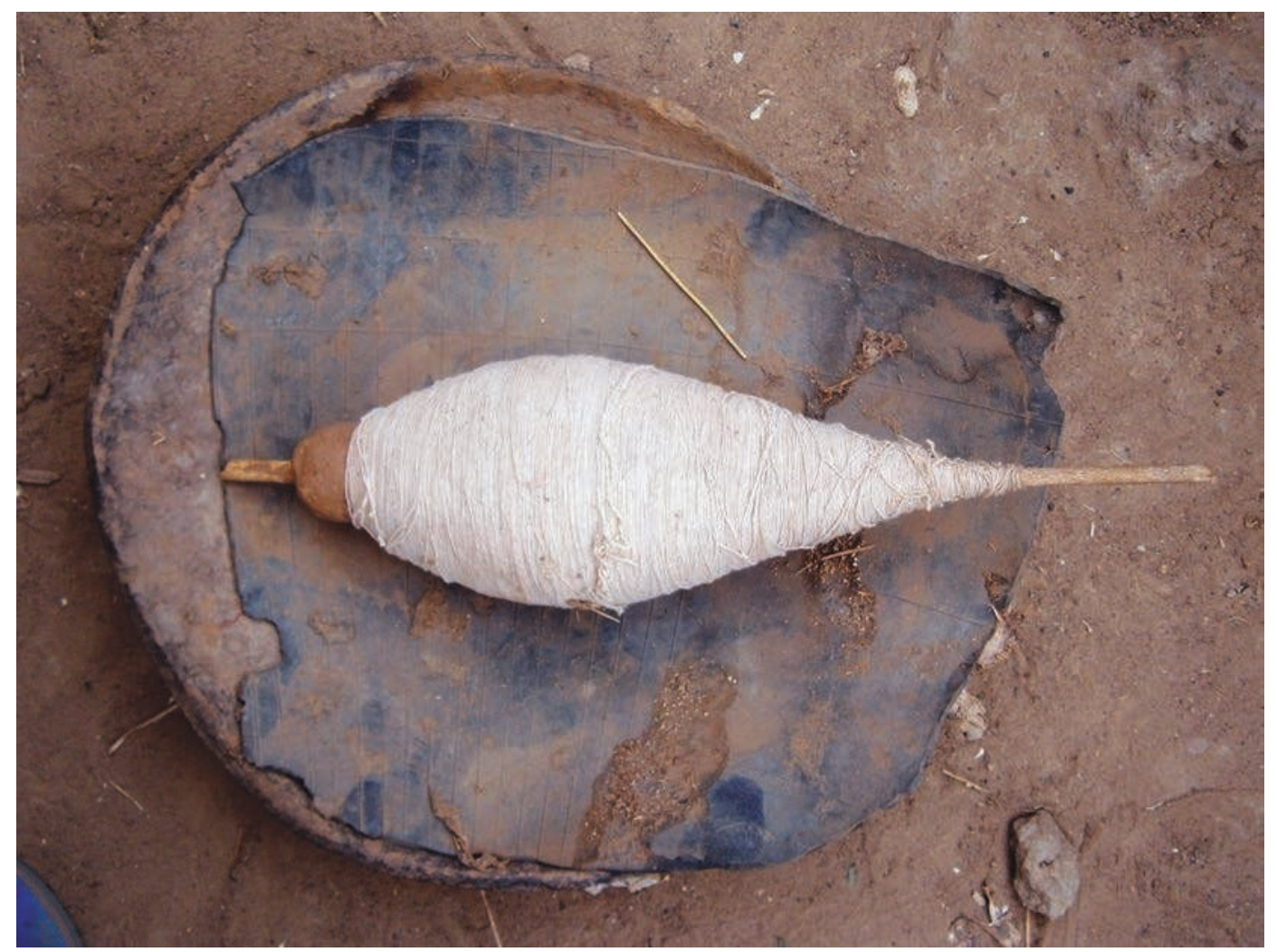

C'est le présent qui façonne son passé et non l'inverse, remarquait Lenclud (1987) dans un article consacré à l'insaisissable notion de tradition. Par «façonner», il désignait le travail de tri, d'interprétation et de reformulation auquel se livre toute société soucieuse de faire correspondre le passé à ses aspirations contemporaines. Un tel travail est inlassablement mis en valeur dans les études consacrées à la «biographie» ou «trajectoire sociale» des objets (Bonnot 2002, Hahn $\&$ Weiss 2013, Kopytoff 1986, Wateau et al. 2011). On y voit comment des choses du passé sont sélectionnées et mises en scène pour permettre à des individus de se «dire» aux autres (par ex. Sizaire \& Petit 2009) -à la manière du faux catalogue de vente de la plasticienne Leanne Shapton (2009) - et se transforment aux plans physique et représentationnel lors du passage d'un contexte sociohistorique à un autre.

Si ces façons de conjuguer le passé au présent apparaissent également dans le Dendi béninois, ce n'est pas elles que nous avons souhaité aborder ici. L'examen des restes d'une ancienne filière textile révèle en effet un tout autre phénomène: le surgissement d'éléments du passé dans le présent - souvent à l'insu des vivants et parfois contre leur gré. C'est à ce titre que nous avons choisi de parler de «fantômes. » Face à eux, l'ethnographe - chasseur de fantômes débutant - perçoit initialement une inquiétante anarchie : aucune matérialité partagée, pas de principes directeurs 
communs, des situations d'apparition disparates, des effets contrastés... Cette impression de fouillis est renforcée par l'inexpérience et le recours habituel aux dialectiques rupture/continuité, passé/présent, ici/là-bas dans l'approche biographique des faits matériels.

Intégrer les fantômes dans le projet ethnographique et s'intéresser à la façon dont ils cohabitent avec les vivants permet d'échapper à un cadre analytique trop étriqué, tout en développant une attention pour d'autres catégories de faits. Plutôt que se focaliser sur des éléments saillants - en raison du maintien en vie d'une activité ou de l'existence de processus de requalification ou de patrimonialisation - une place est accordée à des catégories intermédiaires, dont nous avons vu que la discrétion ou l'occultation temporaire ne préjuge en rien la pertinence. Prêter attention aux restes fantômes permet aussi d'établir une cartographie plus consistante de l'activité disparue. On multiplie les plis et les prises (Legrain 2009) par lesquels l'expérience des anciens praticiens peut être approchée plus intimement - nous montrerons dans un prochain texte comment nous avons progressivement apprivoisé et «travaillé » les fantômes évoqués ici, afin de reconstituer l'ancienne filière textile du Dendi. Enfin, les situations de «surgissement» des fantômes attirent l'attention sur un aspect essentiel de la technique : l'attachement au métier et à certaines de ses matérialisations.

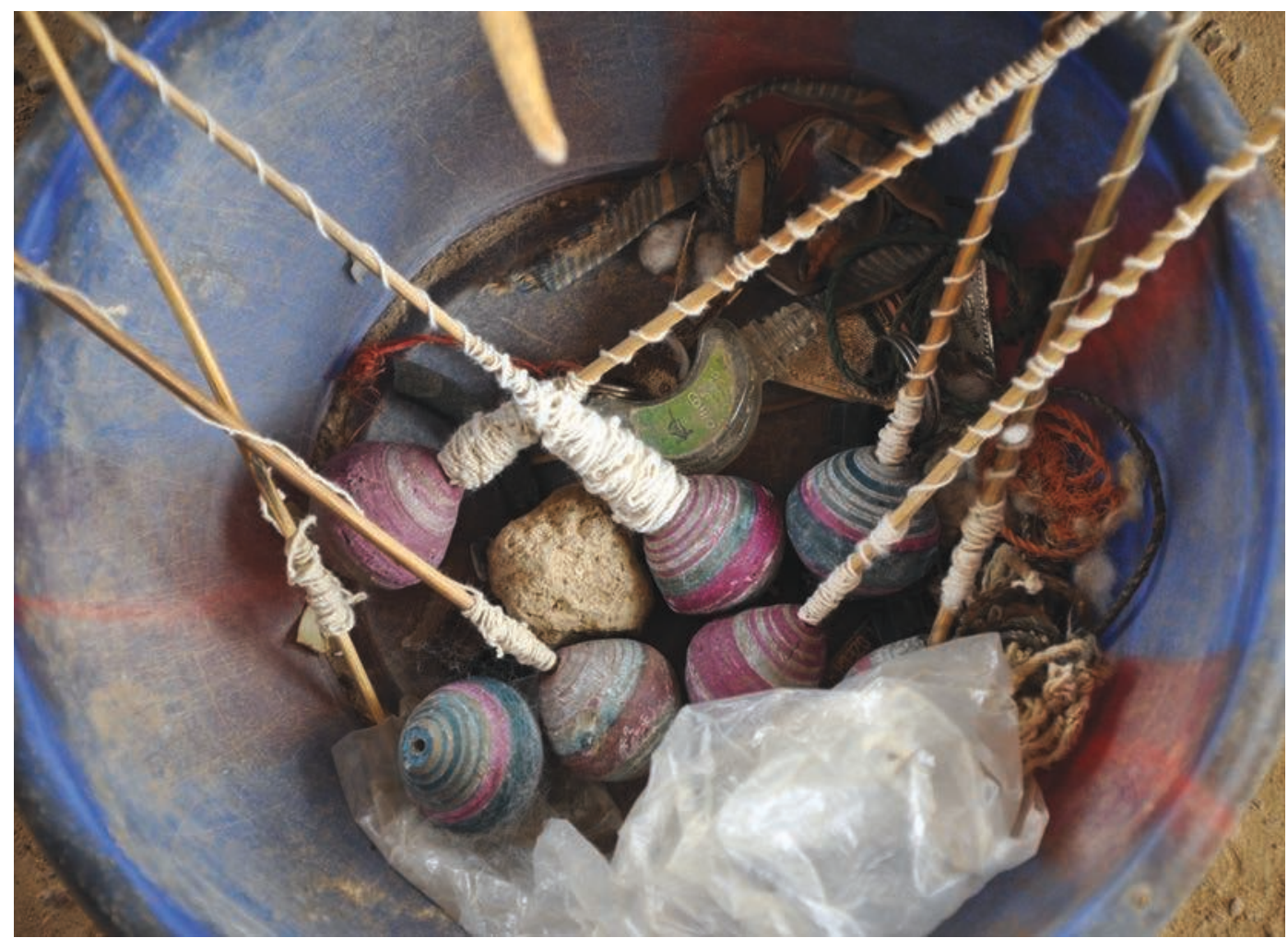

11. Les fuseaux. Ces objets emblématiques de la chaîne opératoire du tissage, traînent encore dans les chambres des vieilles femmes. Pas de stratégie de conservation, pas de projet mémoriel, seul l'attachement impassible de l'ancienne fileuse en assure la conservation. 
Les lieux et dispositifs particuliers dans lesquels ils s'ancrent sont à cet égard des sites d'observation privilégiés, car ils permettent autant de cerner les conditions d'émergence de cet attachement, que d'en envisager l'évolution, entre apparitions et léthargie.

\section{Notes}

1. Ce projet dirigé par Anne Haour (University of East Anglia) a bénéficié d'un financement du European Research Council, Septième programme-cadre (FP/2007-2013) / ERC Grant Agreement 263747

2. Birnin Lafiya, 19 février 2011.

3. Karimama, 20 février 2011.

4. Suivant les informations collectées par entretien, de quelques décilitres à plus de 30 ou 40 .

5. Torozougou, 6 février 2013.

6. Plusieurs chansons destinées aux tisserands commencent par la phrase «Tisserand Ô Tisserand ». La chanson à laquelle notre interlocuteur fait référence met l'accent sur l'ascendance des deux parties: «Tisserand Ô Tisserand / Mon père n'est pas tisserand / Toi et moi, nous allons danser».

8. Cette danse imite le mouvement de balancier des bras du tisserand lorsqu'il lance sa navette.

9. Lactivité de teinture à l'indigo a en effet été menée dans une dizaine d'autres villages du Dendi jusque dans les années 1970-1980. Contrairement à Karimama, ces ateliers ont depuis été recouverts par des constructions.

10. Mamassi Gourma, 22 janvier 2013.

11. Kompanti Débéré, 31 janvier 2013.

12. Torozougou, 6 janvier 2013.

13. Tondi Banda, 31 janvier 2015.

14. Tondi Banda, 31 janvier 2015.

15. Toumboutou, 12 mars 2015.

16. Kompa, 31 janvier 2012.

17. Karimama, 2 février 2012.

7. Pékinga, 27 janvier 2013.

\section{Les auteurs}

Olivier P. Gosselain est professeur dans le département d'Histoire, Art et Archéologie de l'Université libre de Bruxelles. Ses recherches portent sur les artisanats, les cultures matérielles et l'histoire en Afrique de l'Ouest. Il a récemment participé au projet ERC «Crossroads of Empires ».

Lucie Smolderen est membre du CReA-Patrimoine (Université libre de Bruxelles, aspirante F.R.S.-FNRS). Ses recherches doctorales portent sur l'histoire des activités féminines du Dendi (Nord Bénin).

\section{Iconographie}

Image ouverture. Kompa, le 31.01.2012 : Au détour d'une rue, dans le village de Kompa une femme nous montre une bobine de fil qu'elle conserve depuis plusieurs années. (C) L. Smolderen.

\section{Références}

Bonnot, Th. 2002 La Vie des objets. D’ustensiles banals à objets de collection. Paris: Éditions de la Maison des Sciences de L'Homme.

Bornand, S. 2011 «La construction d'une mémoire partagée». Cahiers de littérature orale 69: 107-139.

$1,2,4,5,6,8$ et 11 . (C) O. P. Gosselain. $3,7,9$ et 10 . (C) L. Smolderen.

Gell, A. 1992 «The Technology of Enchantment and the Enchantment of Technology » in J. Coote et A. Shelton dir. Anthropology, Art, and Aesthetics: 40-63. Oxford: Clarendon Press. 
Gosselain, O.P. 2015 «Roads, Markets, Migrants. The Historical Trajectory of a Male Hausa Pottery Tradition in Southern Niger » in W. Gauß, G. Klebinder-Gauß $\&$ C. von Rüden dir. The Distribution of Technological Knowledge in the Production of Ancient Mediterranean Pottery. Vienne: Sonderschriften ÖAI : 277-296.

Hahn H. P. \& H. Weis dir. 2013 Mobility, Meaning, and Tranformations of Things: Shifting Contexts of Material Culture Through Time and Space. Oxford: Oxbow Books.

Haour,A., Banni Guené, O., Gosselain, O.P. \&A. Livingstone Smith 2011 «Survey Along The Niger River Valley at the Bénin-Niger Border ». Nyame Akuma 76: 23-32.

Hénnion, A. 1988 Comment la musique vient aux enfants? Une anthropologie de l'enseignement musical. Paris: Anthropos.

Jacomy, B. 2002 L'Âge du plip. Chroniques de l'innovation technique. Paris: Éditions du Seuil.

Julien, M.-P. \& C. Rosselin dir. 2009 Le Sujet contre les objets... tout contre. Ethnographies de cultures matérielles. Paris: Éditions du CTHS.

Kopytoff, I. 1986 «The Cultural Biography of Things: Commodization as a Process $»$ in A. Appadurai dir. The Social Life of Things. New York: Cambridge University Press: 64-91.

Lave, J. \& E. Wenger 1991 Situated Learning. Legitimate Peripheral Participation. Cambridge: Cambridge University Press.

Legrain, L. 2009 «L'Art de se faire surprendre», Techniques ECulture 52-53: 258-281.

Lenclud, G. 1987 «La Tradition n'est plus ce qu'elle était. Sur les notions de tradition et de société traditionnelle en ethnologie», Terrain 9: 110-123.

Naji, M. 2009 «La Formation des féminités à travers le tissage dans le Sirwa (Maroc)», in M.-P. Julien $\&$ C. Rosselin dir. Le Sujet contre les objets... tout contre.
Ethnographies de cultures matérielles. Paris: Éditions du CTHS : 243-263.

Olivier de Sardan, J.-P. 1984 Les Sociétés songhay-zarma (Niger-Mali). Paris : Karthala.

- 1982 Concepts et conceptions songhay-zarma. Paris: Nubia.

Ravenhill, P.L. 1991 «Instruction publique, collections nationales et connaissance des musées en Afrique» in I. Serageldin \&J. Taboroff dir. Culture et développement en Afrique. Washington: Banque Mondiale: 197-310.

Shapton, L. 2009 Pièces importantes et effets personnels de la collection Lenore Doolan et Harold Morris, comprenant livres, prêt-à-porter et bijoux. Paris: Éditions de l'Olivier.

Sizaire, V. \& P. Petit 2009 «Ce que disent les objets: réflexion sur la mémoire populaire à Lubumbashi » in I. Ndaywel E Nziem \& E. Mudimbe-Boyi dir. Images, mémoires et savoirs. Une histoire en partage avec Bogumil Koss Jiewsiewicki. Paris: Karthala: 155-176.

Smolderen, L. 2013 La filière artisanale du coton dans le Dendi et le Borgou (Nord Bénin). Un fait ethnographique et historique. Mémoire de Master, Université libre de Bruxelles.

Smolderen, L. \& R. Minguet 2014 «Un Fil d'Ariane dans le Dendi (Nord Bénin)», TechniquesE Culture 61: 304-317.

Tamari, T., 1997 Les Castes de l'Afrique occidentale. Artisans et musiciens endogames. Nanterre: Société d'Ethnologie.

Vallard, A. 2012 «De la Jupe à la femme. Tissage, vêtement et subjectivation au Laos» in E. Anstett \& M.-L. Gélard dir. Les objets ont-ils un genre? Culture matérielle et production sociale des identités sexuées. Paris: Armand Colin: 105-120.

Wateau, F., Perlès, C. \& P. Soulier 2011 Profils d'objets. Approches d'anthropologues et d'archéologues. Paris: De Boccard.

\section{Pour citer cet article}

Gosselain O.P. \& L. Smolderen 2016 «Les fantômes du Dendi. Lorsque surgissent les restes d'une ancienne filière textile au Nord Bénin », TechniquesECulture 65-66 « Réparer le monde. Excès, reste et innovation», p. 452-469. 